\title{
Molecular Cloning and Functional Characterisation of a Polyunsaturated Fatty Acid Elongase in a Marine Bivalve Crassostrea angulata
}

\author{
Hongkuan Zhang ${ }^{1,2}$, Helu Liu ${ }^{1,2}$, Dewei Cheng ${ }^{1,2}$, Hongxing Liu ${ }^{1,2}$, Huaiping Zheng ${ }^{1,2, *}$ \\ ${ }^{1}$ Key Laboratory of Marine Biotechnology of Guangdong Province, Shantou University, Shantou, China \\ ${ }^{2}$ Mariculture Research Center for Subtropical Shellfish \& Algae, Guangdong Province, Shantou, China \\ *Corresponding author: hpzheng@stu.edu.cn
}

\begin{abstract}
The elongases of fatty acids (ELO) are essential for long chain polyunsaturated fatty acid (LC-PUFA) biosynthesis, and their activities depend on the substrates. The full length cDNA of Crassostrea angulata ELO (CaELO) was cloned by RACE PCR and its function was confirmed. The CaELO encodes a polypeptide of 309 amino acid residues, which containes a histidine box HXXHH motif conserved in all elongases and shares high similarity to the elongases of Chlamys nobilis and Octopus vulgaris. Phylogenetic analysis showed that the putative elongase was placed in the same group with ELOVL2 and ELOVL5, which have been demonstrated to be critical enzymes participating in the biosynthesis of PUFAs in vertebrates. When expressed in Saccharomyces cerevisiae, CaELO was able to elongate n-3 and n-6 PUFA substrates with chain lengths of C18 and C20, indicating that the CaELO had similar substrate specificities to vertebrate ELOVL5. CaELO had lower activity to elongate monounsaturated fatty acids, but had not activity to saturated fatty acids. Interestingly, the conversion rate of PUFAs depended on the length of carbon chain, the number of double bond, and n-3 / n-6 series in the species.
\end{abstract}

Keywords: PUFA, ELOVL, Crassostrea angulata, Biosynthesis

Cite This Article: Hongkuan Zhang, Helu Liu, Dewei Cheng, Hongxing Liu, and Huaiping Zheng, "Molecular Cloning and Functional Characterisation of a Polyunsaturated Fatty Acid Elongase in a Marine Bivalve Crassostrea angulata." Journal of Food and Nutrition Research, vol. 6, no. 2 (2018): 89-95. doi: 10.12691/jfnr-6-2-4.

\section{Introduction}

Polyunsaturated fatty acids (PUFAs) are important components of cellular structure and function and serve as precursor to eicosanoids, including prostaglandins and leukotrienes. These eicosanoids have a number of functions in animals, involving in inflammatory responses, regulation of blood pressure, and reproductive function [1]. PUFAs, such as linoleic acid (LA, 18:2n-6) and $\alpha$ linolenic acid (ALA, 18:3n-3) which are essential fatty acids for biosynthesis of long-chain polyunsaturated fatty acids (LC-PUFA), cannot be endogenously biosynthesized by human and so must be obtained from diet.

In animals, the ability to produce PUFA varies and is dependent on their complement of fatty acyl desaturase and elongases. In vertebrates, the pathway of biosynthesis LC-PUFAs has been clearly described [2], which involves sequential desaturation and elongation of essential PUFA precursors, LA and ALA. While in molluscs, fatty acyl elongase genes with differing fatty acid (FA) substrate specificities are only described in Chlamys nobilis and Octopus vulgaris $[3,4,5]$.

Microalgae, the major source of LA, ALA, C20 and C22 PUFAs, are the main feed for the filter-feeder bivalves $[6,7]$. Previous studies have showed that these
PUFAs are crucial to the survival, growth, development and reproduction for molluscs, such as Mytilus galloprovincialis [8], Babylonia areolata [9], Solen marginatus [10], and Ostrea angasi [11], etc. Decades of research on PUFAs of elongation and desaturation activity in bivalves has improved our understanding of the PUFAs synthesis pathway. In the early studies, by using labeled PUFA precursors, clam Mesoderma mactroides and Pacific oyster Crassostrea gigas were demonstrated to have the ability to desaturate and elongate PUFA precursors [12,13]. In addition, Scapharca broughtoni and Mytilus edulis were reported to have the capabilities of de novo synthesis for some peculiar fatty acids (FAs) called nonmethylene interrupted fatty acids (NMIs) [14,15]. More importantly, in recent years, our lab has provided the first compelling evidence that $C$. nobilis can de novo biosynthesize 20:5n-3 and 20:4n-6 from PUFA precursors though the " $\Delta 8$ pathway" [16].

The Crassostrea oysters are important edible marine bivalves, which have been cultured worldwide. It is known that oysters are rich in PUFAs $[17,18]$, and part of them obtained from their diets. However, whether they have the ability to biosynthesize PUFAs themselves is still unknown.

In the present study, the cDNA of fatty acyl elongase was isolated in the Crassostrea angulata, and function of the encoded polypeptide was characterized in yeast 
Saccharomyces cerevisiae to investigate whether the gene could elongate PUFA substrates.

\section{Materials and Methods}

\subsection{Cloning of Putative PUFA Elongase from C. angulata}

The adult oysters used in the present study were sampled from a cultured population at Nan'ao Island of Guangdong Province, China. The adductor was sampled and total RNA was extracted using TransZol Up reagent (TransGen Biotech, China). Then, $1 \mu \mathrm{g}$ of total RNA was used as template for cDNA synthesis by using SuperScript III reverse transcriptase PCR and Oligo-dT as primers following the instructions of the manufacturer (Invitrogen). Partial cDNA sequence of elongase was obtained from transcriptome sequencing of $C$. angulate (SRX481252). Then forward primer CAEF and reverse primer CAER (Table.1) were used to confirm the cDNA fragment. PCR amplification was performed under the following PCR conditions: initial denaturation at $95^{\circ} \mathrm{C}$ for $2 \mathrm{~min}, 35$ cycles of denaturation at $95^{\circ} \mathrm{C}$ for $30 \mathrm{~s}$, annealing at $60^{\circ} \mathrm{C}$ for $30 \mathrm{~s}$, and extension at $72^{\circ} \mathrm{C}$ for $90 \mathrm{~s}$. The PCR products were separated on $1 \%$ agarose gel, and then purified with a PCR purification kit (TransGen Biotech). The purified PCR product was ligated with the $\mathrm{p} E A S Y$ - T1 Cloning Kit (TransGen Biotech), and transformed into Trans $5 \alpha$ Chemically Competent Cell (TransGen Biotech). The recombinants were identified through blue-white color selection in ampicillin containing LB plates and screened with M13-47 and RV-M primers, and then sequenced on both strands.

Table 1. Sequence for primers used in the present

\begin{tabular}{|c|c|}
\hline $\begin{array}{l}\text { Primer } \\
\text { name }\end{array}$ & Sequences \\
\hline CAEF & 5'-TACAGCGATGTCAGTGTGGCA-3' \\
\hline CAER & 5'-ACAACGACTTCACCAGAGGAGC-3' \\
\hline CAE51 & 5'-ACGTAGACCGGGTTATCGTACAGCA-3' \\
\hline CAE52 & 5'-GCCCTCTTCATACAGTCTCAACACA-3' \\
\hline CAE31 & 5'-CACACCATCCAGACAACTTTCACGG-3' \\
\hline CAE32 & 5'-AAGACTGACCTAGCTCAAAATGGGG-3' \\
\hline M13-47 & 5'- CGCCAGGGTTTTCCCAGTCACGAC-3' \\
\hline $\begin{array}{l}\text { M13- } \\
\text { RVM }\end{array}$ & 5'-AGCGGATAACAATTTCACACAGGA-3' \\
\hline \multicolumn{2}{|c|}{ Function primers } \\
\hline CAEF-1 & $\begin{array}{l}\text { 5'-CCCAAGCTTATGGAGAAAATTCCAGTTGTTAAA } \\
\text { G-3' }\end{array}$ \\
\hline CAER-1 & 5'-CCGGAATTCTCAATCACTTTTCGAGTGTC-3' \\
\hline \multicolumn{2}{|c|}{ Real-time PCR primers } \\
\hline CAEE-F & 5'-GTTTTGTAGCTCGCTGAATGC-3' \\
\hline CAEE-R & 5'-ACCCGTGAAAGTTGTCTGGAT-3' \\
\hline$\beta$-actin-F & 5'-GCGGCAGTGGTCATCTCCT-3' \\
\hline$\beta$-actin-R & 5'-GCCCTTCCTCACGCTATCCT-3' \\
\hline * Restrict & $\begin{array}{l}\text { tes for Hind III and EcoR I are underlined in primer } \\
\text { AER-1 sequences. }\end{array}$ \\
\hline
\end{tabular}

Based on the partial cDNA sequence of the elongase, the complete CaELOVL cDNA was amplified through 3'RACE cDNA and 5'-RACE PCR with the SMARTerTM RACE cDNA Amplification Kit (Clontech, Mountain View, CA) and LA Taq polymerase (TaKaRa, Japan), using touch-down PCR and nested PCR strategy. The touch-down PCR was carried out in a $50 \mu$ l volume containing $2.5 \mu \mathrm{l}$ 5'-RACE cDNA (or 3'-RACE cDNA) template, $1 \mu$ l CAE51 (or CAE31) primer $\left(10 \mathrm{mmol} \mathrm{L}^{-1}\right)$, $1 \mu \mathrm{l}$ UPM (long UPM $10 \mathrm{mmol} \mathrm{L}^{-1}$, short UPM $10 \mathrm{mmol}$ $\mathrm{L}^{-1}$ ) (Table 1), $0.5 \mu \mathrm{L}$ LA Taq DNA Polymerase ( $\left.5 \mathrm{U} / \mu \mathrm{l}\right), 5$ $\mu l 10 \times$ LA Taq Buffer II ( $\mathrm{Mg}^{2+}$ Plus), $8 \mu \mathrm{l}$ dNTP Mixture (2.5 mM each), and $32 \mu \mathrm{l}$ sterilized ultrapure water. The PCR reaction program was performed as follows: $94^{\circ} \mathrm{C}$ for $2 \mathrm{~min}$ followed by 35 cycles of $94^{\circ} \mathrm{C}$ for $30 \mathrm{~s}, 68^{\circ} \mathrm{C}$ $60{ }^{\circ} \mathrm{C}$ for $30 \mathrm{~s}$ in the initial 9 cycles decrementing $1{ }^{\circ} \mathrm{C}$ /cycle, $60^{\circ} \mathrm{C}$ for $30 \mathrm{~s}$ for the remaining 26 cycles, $72^{\circ} \mathrm{C}$ for $2 \mathrm{~min}$, and a final extension step at $72^{\circ} \mathrm{C}$ for $10 \mathrm{~min}$. The products of touch-down PCR were used as template for the subsequent nested PCR. The expected DNA fragment was separated on a $1 \%$ agarose gel and then purified with a PCR purification kit (TransGen Biotech). Purified DNA fragment was sub-cloned into $\mathrm{p} E A S Y-\mathrm{T} 1$, and then transformed into Trans5a Chemically Competent Cell. Positive recombinant clones were identified by blue-white color selection in ampicillin-containing LB plates and PCR screening with M13-47 and M13-RVM primers, and then sequenced by a commercial company (Sangon Biotech, Shanghai, China). The full length cDNA of CaELOVL was aligned from the overlapping cDNA clones.

\subsection{Phylogenetic Analysis of Putative PUFA Elongase from $C$. angulata}

A phylogenetic tree was constructed on the basis of protein sequence of ELOVL from the putative $C$. angulata ELOVL (CaELOVL) and other organisms, including vertebrate and invertebrate. A multiple sequence alignment was performed using ClustalX2.1 and a phylogenetic tree was made using the neighbor-joining method in MEGA 5.1 package. Confidence in the resulting phylogenetic tree branch topology was measured by bootstrapping through 1000 iterations.

\subsection{Real-time PCR Analysis of CaELOVL Transcript Levels}

Total RNA from different tissues of adductor, gill, gonad and mantle was isolated as described above. Firststrand cDNA was synthesized using RT reagent Kit with gDNA Eraser (Takara, Dalian, China) according to the manufacturer's instructions. Two gene-specific primers were designed to amplify a product of $158 \mathrm{bp}$, which was quantified by real-time RT-PCR with the SYBR ${ }^{\circ}$ Premix Ex Taq ${ }^{\mathrm{TM}}$ II Kit (Perfect Real Time) (Takara, Japan) following the manufacturer's protocol. The reaction was performed in a LightCycler ${ }^{\circledR} 480$ (Roche). A total reaction volume of $20 \mu \mathrm{l}$ containing $10 \mu \mathrm{l}$ SYBR ${ }^{\circledR}$ Premix $\mathrm{Ex} \mathrm{Taq}^{\mathrm{TM}}$ II, $2 \mu$ l the four-fold diluted cDNA, $0.8 \mathrm{ml}$ each primer (CAEE-F and CAEE-R to amply CaELOVL gene, $\beta$-actin-F and $\beta$-actin-R to amply $\beta$-actin, $10 \mathrm{mmol} \mathrm{L}^{-1}$, Table 1) and $6.4 \mu \mathrm{l}$ ultra-pure water was carried out following a denaturation step of $95^{\circ} \mathrm{C}$ for $30 \mathrm{~s}, 45$ cycles of $95^{\circ} \mathrm{C}$ for $5 \mathrm{~s}, 60^{\circ} \mathrm{C}$ for $30 \mathrm{~s}$, a melting curve analysis from $65^{\circ} \mathrm{C}$ to $95^{\circ} \mathrm{C}$ and a cooling step of $40^{\circ} \mathrm{C}$ for $10 \mathrm{~min}$. Each sample was run in triplicate. Data were analyzed by using the LightCycler480 software (Roche) after the PCR program. Relative mRNA expression level of CaELOVL 
was determined with the $2^{-\Delta \Delta^{\mathrm{Ct}}}$ algorithm with $\beta$-actin from C. angulata as the internal control.

\subsection{Heterologous Expression of Elongase ORFs in Yeast}

Expression primers were designed for PCR cloning putative elongase cDNA ORF. The forward primer and the reverse primer was CAEF-1, contained a HindIII site (underlined), and CAER-1, contained an EcoRI site (underlined). PCR was performed using TransStart ${ }^{\circledR}$ FastPfu DNA Polymerase (Trans GenBiotech) following the manufacturer's instructions. Amplification involved an initial denaturation step at $95^{\circ} \mathrm{C}$ for $2 \mathrm{~min}$, followed by 30 cycles of denaturation at $95^{\circ} \mathrm{C}$ for $20 \mathrm{~s}$, annealing at $56^{\circ} \mathrm{C}$ for $30 \mathrm{~s}$, and extension at $72^{\circ} \mathrm{C}$ for $60 \mathrm{~s}$. After PCR, the DNA fragments were cut with HindIII/EcoRI and ligated into the similarly digested yeast expression vector pYES2.0 (Invitrogen), which was then used to transform DH5 $\alpha$ E. coli competent cells. Transformation of yeast with recombinant plasmids and yeast culture were carried out using the S.c. EasyComp Transformation Kit (Invitrogen Ltd.). Selection of yeast containing the elongase/pYES2 constructs was on S. cerevisiae minimal medium minusuracil (SCMM-uracil). Culture of the recombinant yeast was carried out in SCMM-uracil broth as described previously [19], using galactose induction of gene expression. Each culture was supplemented with one of the following PUFA substrates: C18:2n-6, C18:3n-3, C20:4n-6,C20:4n-6, C20:5n-3 and C22:3n-6. Yeast cells were harvested, washed, and dried, and then lipid was extracted by homogenization in chloroform/methanol (2:1, $\mathrm{vol} / \mathrm{vol}$ ) containing $0.01 \%$ butylated hydroxytoluene (Sigma, Ltd) as antioxidant. FAMEs were prepared, extracted, purified by TLC on $20 \mathrm{~cm} \times 20 \mathrm{~cm} \times 0.25 \mathrm{~mm}$ silica gel 60 plate (Merck, Germany), and analyzed using gas chromatography (GC-17A, Shimadzu, Kyoto, Japan) equipped with a hydrogen flame ionization detector and a $30 \mathrm{~m} \times 0.25 \mathrm{~mm} \times 0.25 \mu \mathrm{m}$ capillary column (VF-23ms, Varian, USA), all as described below. The column was temperature programmed from 50 to $150^{\circ} \mathrm{C}$ at $6^{\circ} \mathrm{C} / \mathrm{min}$ and 150 to $230^{\circ} \mathrm{C}$ at $5^{\circ} \mathrm{C} / \mathrm{min}$. Hydrogen was used as carrier gas at a flow rate of $1.1 \mathrm{ml} / \mathrm{min}$. The temperature of detector and injection port was set at $250^{\circ} \mathrm{C}$. Individual methylesters were identified by comparison to authentic standards. The proportion of substrate fatty acid converted to the longer chain fatty acid product was calculated from the gas chromatograms as 100.[Product Area / (Product Area + Substrate Area)].

\section{Results}

\subsection{CaELOVL cDNA Sequences and Phylogenetics}

A 2238bp full-length cDNA sequence was obtained by 5'- and 3'-RACE PCR and was deposited in the GenBank database under the accession number KY012771. It contains an ORF of 930bp (including stop codon) encoding a putative protein of 309 amino acid (aa), sharing $57 \%$ and $54 \%$ aa sequence identity with C. nobilis elongase (KF245423) and O. vulgaris (AFM93779.1), respectively. And it has $42-45 \%$ identity to Xenopus tropicalis ELOVL5 (NP001011248), Siganus canaliculatus ELOVL5 (ADE34561), Gallus gallus ELOVL2 (NP001184237), Homo sapiens ELOVL5 (NP068586). The newly cloned fatty acyl elongase protein possessed the typical histidine box HXXHH motif (Figure 1) conserved in all elongases. Seven putative transmembrane-spanning domains were predicted using TMHMM 2.0, five of which (I, II, V, VI, VII) had been reported in a mouse ELOVL2 [20]. A phylogenetic tree was constructed on the basis of the aa sequence alignments between the putative CaELOVL and other ELOVL family members $(2,5)$ from invertebrates, and as well as from vertebrates (Figure 2). The CaELOVL had the closest relationship with ELOVL from C. nobilis, then $O$. vulgaris, and formed a group close to ELOVL2 and ELOVL5 from vertebrates.

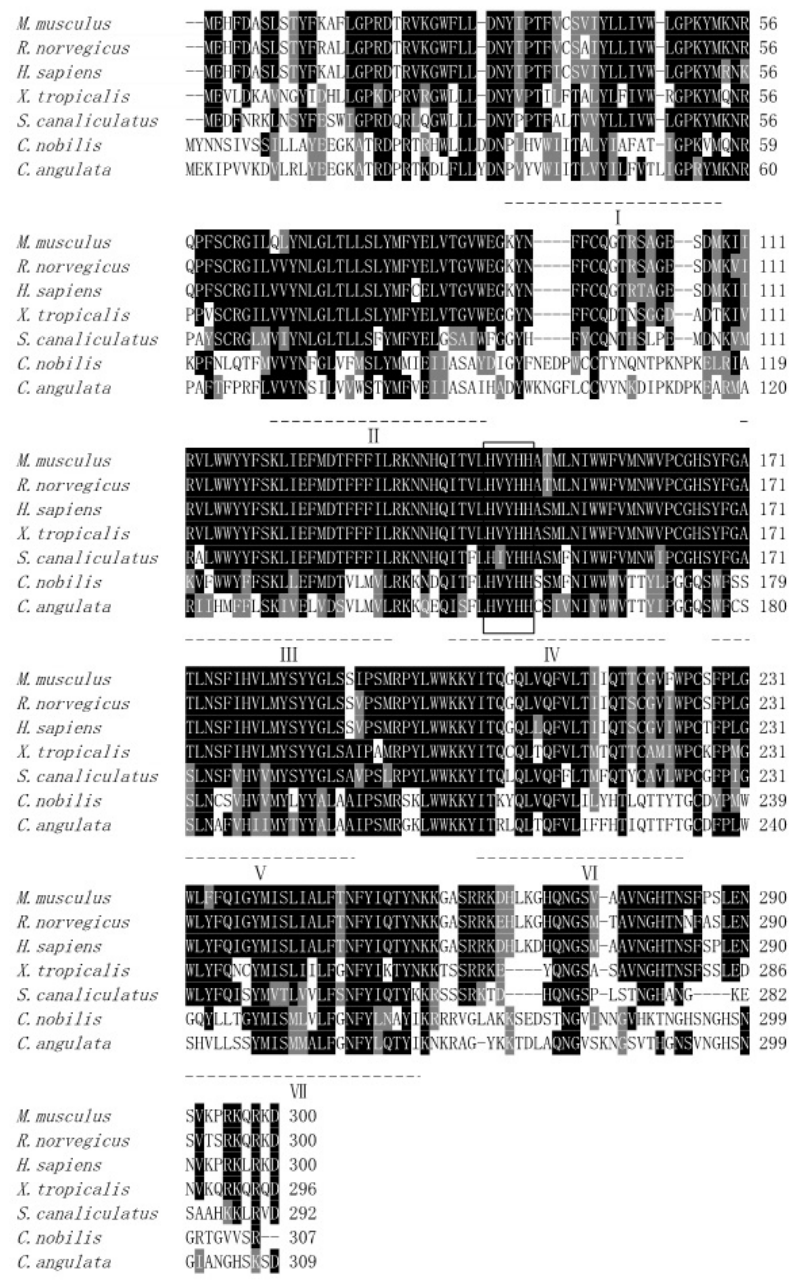

Figure 1. Comparison of the amino acid sequences of PUFA elongases cloning from C. angulata (KY012771) with those from C. nobilis (KF245423), O. vulgaris (AFM93779), Xenopus tropicalis (NP001011248), Siganus canaliculatus (ADE34561), Gallus gallus (NP001184237), and Homo sapiens (NP068586). Identical residues are shaded black and similar residues are gray. The threshold for similarity shading was set at $25 \%$. Indicated is the conserved histidine box motif. Seven (I-VIII) putative membrane-spanning domains (underlined with dashed line) were identified using TMHMM 2.0, five of which (I, II, V, VI, VII) were predicted by Tvrdik et al. (2000) 


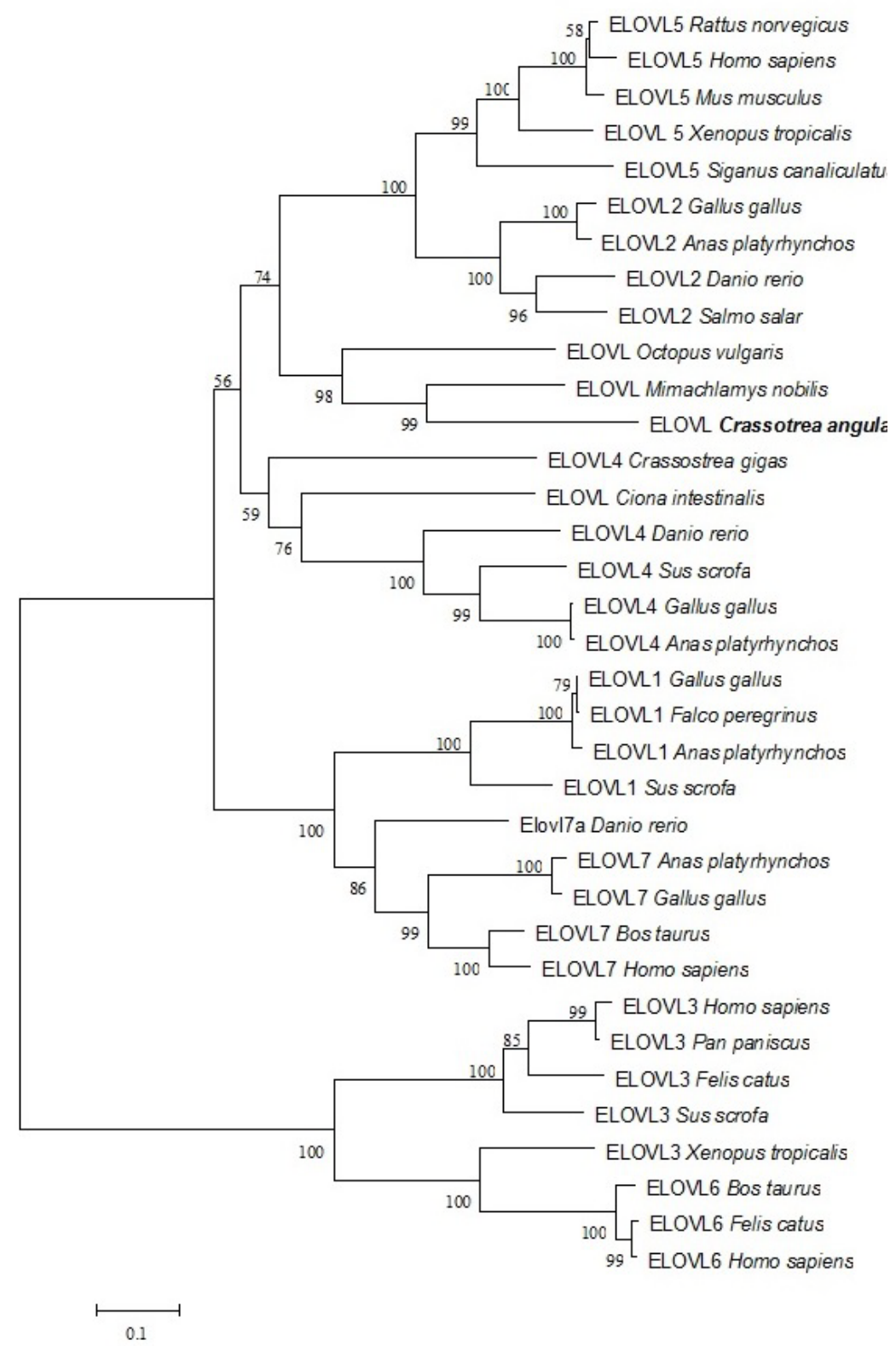

Figure 2. Phylogenetic tree comparing the deduced amino acid sequence of the $C$. angulata elongase of very long-chain fatty acids with a series of protein sequences including representatives of the ELOVL subtypes from other organisms. A multiple sequence alignment was performed using ClustalW and a phylogenetic tree was made using the neighbor-joining method in MEGA 4.1 package. Numbers represent the frequencies with which the tree topology presented was replicated after 1000 bootstrap iterations

\subsection{Tissue Distribution of CaELOVL}

CaELOVL transcripts were detected in all tested tissues (Figure 3), and the level was significantly different among the tissues $(P<0.05)$. And there was a significantly higher level in gill and mantle than in gonad and adductor.

\subsection{Functional Characterization}

Yeast transformed with vector containing no insert contained only four endogenous fatty acids (C16:0, C16:1n-7, C18:0, and C18:1n-9) [19], together with whichever exogenous FA was added. The results were consistent with the well-established observations of lack of PUFA elongase activity in $S$. cerevisiae [21,22]. The FA compositions of yeast transformed with the pYES2ELOVL construct and grown in the presence of different substrates were shown in Figure 4.

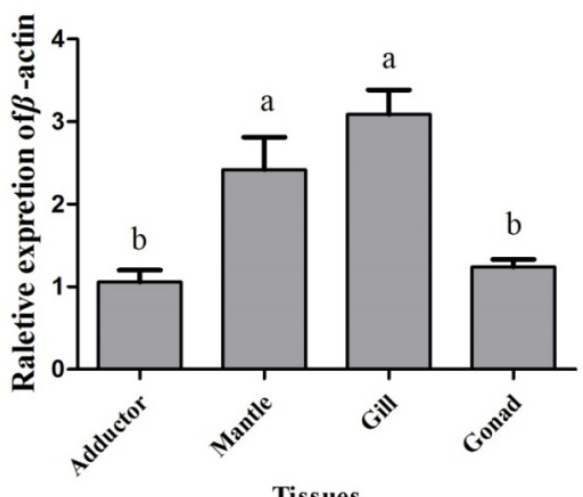

Tissues

Figure 3. Tissue distributions of CaELOVL in $C$. angulata by qRT-PCR analysis. Expression levels in all tissues are presented relative to $\beta$-actin. Vertical bars represent \pm S.D. $(\mathrm{N}=4)$ for each tissue 

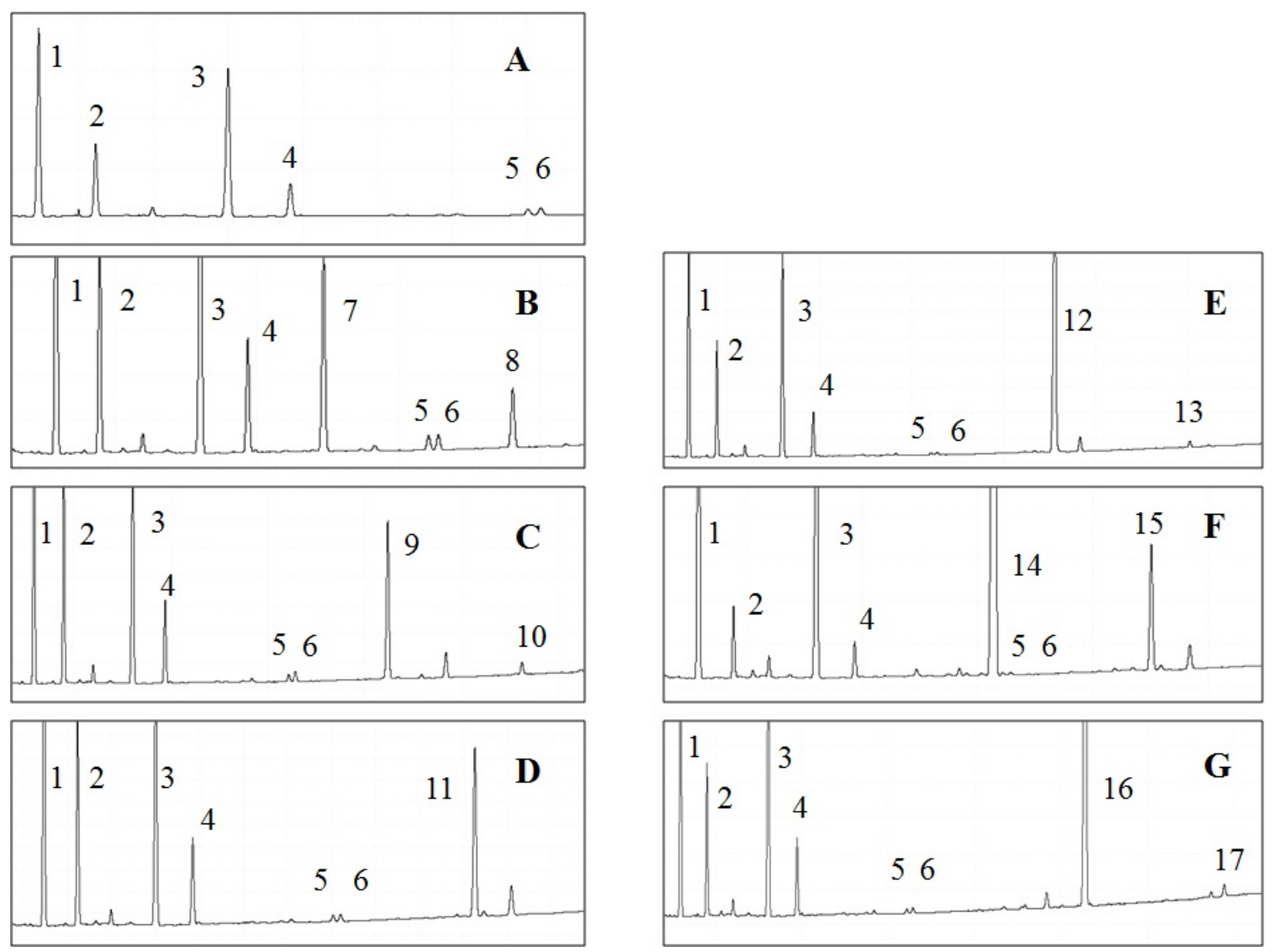

Figure 4. Functional characterization of CaELOVL in transgenic $S$. cerevisiae grown in the presence of fatty acids (FAs). A, B, C, D, E, F and G represent control and adding FA substrate of C18:2n-6, C20:3n-6, C22:3n-6, C20:4n-6, C18:3n-3 and C20:5n-3, respectively. Fatty acids were extracted from yeast transformed with pYES2 vector containing the ORF of the putative fatty acyl elongase cDNA as an insert. Peaks $1-4$ represent the main endogenous FAs of $S$. cerevisiae, namely C16:0 (1), C16:1n-7 (2), C18:0 (3) and C18:1n-9 (4). The remaining main additional peaks (6-17) correspond to the exogenously added FAs and the products of their elongation-C18:2n-6 (7), C20:2n-6 (8), C20:3n-6 (9), C22:3n-6 (10), C22:3n-6 (11), C20:4n-6 (12), C22:4n-6 (13), C18:3n-3 (14), C20:3n-3 (15), C20:5n-3 (16) and C22:5n-3(17). Other minor peaks are 20:1n-9 (5) and 20:1n-7 (6), the latter two resulting from the elongation of $18: 1 \mathrm{n}-9$ and $18: 1 \mathrm{n}-7$.

All substrates including B (C18:2n-6), C (20:3n-6), E (C20:4n-6), F (C18:3n-3), and G (C20:5n-3) had their corresponding products except for D (C22:3n-6). Moreover, we found that conversion rate is significantly different $(P<0.05)$, varying from $25 \%$ to $0.81 \%$ (Table 2).

\section{Discussion}

Polyunsaturated fatty acids (PUFAs), particularly 18- to 22-carbon PUFAs, have obtained a high-profile position in the biomedical and nutraceutical areas because of their specific therapeutic roles in certain clinical conditions. Moreover, public awareness on eating healthy has also brought these PUFAs to the attention of the consumer.

In the past decades, the synthesis pathways of important PUFAs have been clearly described in vertebrates $[23,24]$. As the second largest Phylum in animal kingdom, mollusks have provided delicious and health food for human due to their enriched higher protein and PUFAs $[23,25,26]$. However, mechanism of their enrichment in PUFAs has not been clearly described, although there have been a few reports about biosynthesis PUFAs mechanism in an octopus [4,5] and a bivalve [27] in recent years.

In the present study, the activity of CaELOVL was found similar to that of ELOVL5 more than that of
ELOVL2, which involves specifically in the elongation of $\mathrm{C} 18$ and $\mathrm{C} 20 \mathrm{FAs}$, respcetively. The result is consistent to the previous study in the mollusks [4,27] and in the vertebrates $[24,28]$.

In the past years, bivalves have been regarded as having no or limit ability to biosynthesize n-3 and n-6 long chain PUFAs by elongation and desaturation of the precursors 18:2n-6 and 18:3n-3 [17,29]. Instead bivalves have been thought to obtain the PUFAs from their diets [30,31]. However, in the present study, the oyster was identified to have the ability to biosynthesize PUFAs by itself, and previous study in two mollusks of $O$. vulgaris [4] and $C$. nobilis [16] had the same findings, respectively. Therefore, we conclude that marine mollusks have the ability to biosynthesize PUFAs by themselves.

More interestingly, from the results listed in Table 2, we found that the conversion rate of PUFAs was related to n-3 / n-6 substrates. Significantly higher conversion rate was found with n-6 PUFA substrates than homologous n-3 substrates; however, in vertebrates, most of functionally characterized PUFA biosynthesis genes/enzymes show more activity toward n-3 PUFA substrates [32-35]. Marine microalgae enrich in n-3 series PUFAs [30], the oyster can obtain n-3 PUFAs by feeding these microalgae, this probably explains why the synthesizing ability of the n-3 series PUFAs became weaker than that of the $n-6$ series PUFAs. This might be an adaption to environment in the evolution of animals. 
Table 2. Conversion rate of the CaELOVL for different substrates*.

\begin{tabular}{cccc}
\hline FA substrates & Product & Conversation rate $(\%)$ & Activity \\
\hline $18: 2 \mathrm{n}-6$ & $20: 2 \mathrm{n}-6$ & $25.08 \pm 1.6^{\mathrm{a}}$ & $18 \rightarrow 20$ \\
$18: 3 \mathrm{n}-3$ & $20: 3 \mathrm{n}-3$ & $6.51 \pm 0.64^{\mathrm{b}}$ & $18 \rightarrow 20$ \\
$20: 3 \mathrm{n}-6$ & $22: 3 \mathrm{n}-6$ & $6.84 \pm 0.53^{\mathrm{b}}$ & $20 \rightarrow 22$ \\
$20: 4 \mathrm{n}-6$ & $22: 4 \mathrm{n}-6$ & $1.48 \pm 0.12^{\mathrm{c}}$ & $20 \rightarrow 22$ \\
$20: 5 \mathrm{n}-3$ & $22: 5 \mathrm{n}-3$ & $0.82 \pm 0.06^{\mathrm{c}}$ & $20 \rightarrow 22$ \\
$22: 3 \mathrm{n}-6$ & $24: 3 \mathrm{n}-6$ & 0 & $22 \rightarrow 24$ \\
\hline
\end{tabular}

* Results are expressed as a percentage of total fatty acid substrate converted to elongated product (means $\pm \mathrm{SD}, \mathrm{n}=4$ ). Different letters indicate that means are significantly different as determined by one-way ANOVA followed by Tukey's multiple comparison tests.

In vertebrates, ARA and EPA are biosynthesised from the dietary essential C18 PUFA 18:2n-6 and C18:3n-3 through two alternative pathways, the 'classical' $\Delta 6$ pathway $(\Delta 6$ desaturation $\rightarrow$ elongation $\rightarrow \Delta 5$ desaturation), or alternatively through the so-called ' $\Delta 8$ pathway' (elongation $\rightarrow \Delta 8$ desaturation $\rightarrow \Delta 5$ desaturation). In the present, $C 20: 2 n-6$ and $C 20: 3 n-3$ can be biosynthesised from the dietary essential 18:2n-6 and C18:3n-3, namely 18:2n-6 $\rightarrow$ 20:3n-6 and C18:3n-3 $\rightarrow$ C20:3n-3 for the $\Delta 8$-pathway in $C$. angulata. Although gene responsible for elongation step of this pathway has now been identified in $C$. angulata, no Fad cDNA with $\Delta 5$ and $\Delta 8$-desaturase activity has yet been identified and, consequently, it remains unclear whether the $C$. angulata can biosynthesise ARA and EPA from the dietary essential 18:2n-6. However, in C. nobilis, it has been confirmed that $C$. nobilis could efficiently biosynthesis ARA and EPA from 18:2n-6 and C18:3n-3through ' $\triangle 8$ pathway' [27], based on these results, we speculated that the alternative " $\Delta 8$-pathway" may be the PUFA biosynthesis pathway in C. angulata.

In conclusion, the oyster Crassostra angulata has the ability to biosynthesize PUFAs by itself. And it has a significantly higher conversion rate in $n-6$ PUFA substrates than homologous n-3 substrates. Moreover, we speculated that the alternative " $\Delta 8$-pathway" may be the PUFA biosynthesis pathway in C. angulata.

\section{Acknowledgements}

We are very grateful to Dr. Chiju Wei (Multidisciplinary Research Center, Shantou University) for his careful revision and many constructive comments. Funding for this research was provided by China Modern Agro-industry Technology Research System (CARS-48), Guangdong Province Natural Sciences Foundation (2015A030311027), Science \& Technology Plan (2015B090903081) and Yangfan Plan (14600706), China.

\section{References}

[1] Vance, J. E., Vance, D. E. (Eds.). 2008. Biochemistry of lipids, lipoproteins and membranes. Elsevier.

[2] Hastings, N., Agaba, M., Tocher, D. R., Leaver, M. J., Dick, J. R., Sargent, J. R., \& Teale, A. J. 2001. A vertebrate fatty acid desaturase with $\Delta 5$ and $\Delta 6$ activities. PNAS USA, 98(25), 14304-14309.
[3] Liu, H., Zheng, H., Wang, S., Wang, Y., Li, S., Liu, W., \& Zhang, G. 2013. Cloning and functional characterization of a polyunsaturated fatty acid elongase in a marine bivalve noble scallop Chlamys nobilis Reeve. Aquaculture, 416, 146-151.

[4] Monroig, O., Guinot, D., Hontoria, F., Tocher, D. R., \& Navarro, J. C. 2012. Biosynthesis of essential fatty acids in Octopus vulgaris (Cuvier, 1797): Molecular cloning, functional characterisation and tissue distribution of a fatty acyl elongase. Aquaculture, 360, 45-53.

[5] Monroig, Ó., Tocher, D. R., \& Navarro, J. C. 2013. Biosynthesis of polyunsaturated fatty acids in marine invertebrates: recent advances in molecular mechanisms. Mar Drugs, 11(10), 3998-4018.

[6] Bellou, S., Baeshen, M. N., Elazzazy, A. M., Aggeli, D., Sayegh, F., \& Aggelis, G. 2014. Microalgal lipids biochemistry and biotechnological perspectives. Biotechnol Adv, 32(8), 1476-1493.

[7] Maadane, A., Merghoub, N., Ainane, T., El Arroussi, H., Benhima, R., Amzazi, S., \& Wahby, I. 2015. Antioxidant activity of some Moroccan marine microalgae: Pufa profiles, carotenoids and phenolic content. J Biotechnol, 215, 13-19.

[8] Pettersen, A. K., Turchini, G. M., Jahangard, S., Ingram, B. A., \& Sherman, C. D. 2010. Effects of different dietary microalgae on survival, growth, settlement and fatty acid composition of blue mussel (Mytilus galloprovincialis) larvae. Aquaculture, 309(1), 115-124.

[9] Sangsawangchote, S., Chaitanawisuti, N., \& Piyatiratitivorakul, S. 2010. Reproductive performance, egg and larval quality and egg fatty acid composition of hatchery-reared Spotted Babylon (Babylonia areolata) broodstock fed natural and formulated diets under hatchery conditions. Int J Fish Aquaculture, 2(1), 044-048.

[10] Da Costa, F., Nóvoa, S., Ojea, J., \& Martínez-Patiño, D. 2011. Changes in biochemical and fatty acid composition of the razor clam Solen marginatus (Solenidae: Bivalvia) during larval development. Mar Biol, 158(8), 1829-1840.

[11] O'Connor, S., Moltschaniwskyj, N., Bolch, C. J., \& O'Connor, W. 2012. Dietary influence on growth and development of flat oyster, Ostrea angasi (Sowerby, 1871), larvae. Aquac Res, 43(9), 1317-1327.

[12] De Moreno, J. E., Moreno, V. J., \& Brenner, R. R. 1976. Lipid metabolism of the yellow clam, Mesodesma mactroides: 2polyunsaturated fatty acid metabolism. Lipids, 11(7), 561-566.

[13] Waldock, M. J., \& Holland, D. L. 1984. Fatty acid metabolism in young oysters, Crassostrea gigas: polyunsaturated fatty acids. Lipids, 19(5), 332-336.

[14] Zhukova, N. V. 1991. The pathway of the biosynthesis of nonmethylene-interrupted dienoic fatty acids in molluscs. Comp Biochem Physiol. 100B (4), 801-804.

[15] Zhukova, N. V., \& Svetashev, V. I. 1986. Non-methyleneinterrupted dienoic fatty acids in molluscs from the sea of Japan. Comp Biochem Physiol. 83B (3), 643-646.

[16] Liu, H., Zhang, H., Zheng, H., Wang, S., Guo, Z., \& Zhang, G. 2014. PUFA biosynthesis pathway in marine scallop Chlamys nobilis Reeve. J Agr Food Chem, 62(51), 12384-12391.

[17] Chu, F. L., \& Greaves, J. 1991. Metabolism of palmitic, linoleic, and linolenic acids in adult oysters, Crassostrea virginica. Mar Biol, 110 (2), 229-236.

[18] Cruz-Romero, M. C., Kerry, J. P., \& Kelly, A. L. 2008. Fatty acids, volatile compounds and colour changes in high-pressure-treated oysters (Crassostrea gigas). Innov Food Sci Emerg, 9 (1), 54-61.

[19] Hastings, N., Agaba, M., Tocher, D. R., Leaver, M. J., Dick, J. R., Sargent, J. R., \& Teale, A. J. 2001. A vertebrate fatty acid desaturase with $\Delta 5$ and $\Delta 6$ activities. P Natl Acad Sci USA, 98(25), 14304-14309.

[20] Tvrdik, P., Westerberg, R., Silve, S., Asadi, A., Jakobsson, A., Cannon, B. \& Jacobsson, A. 2000. Role of a new mammalian gene family in the biosynthesis of very long chain fatty acids and sphingolipids. J Cell Biol, 149(3), 707-718.

[21] Agaba, M., Tocher, D. R., Dickson, C. A., Dick, J. R., \& Teale, A. J. 2004. Zebrafish cDNA encoding multifunctional fatty acid elongase involved in production of eicosapentaenoic (20: $5 n-3)$ and docosahexaenoic (22: 6n-3) acids. Mar Biotechnol, 6(3), 251-261.

[22] Morais, S., Monroig, O., Zheng, X., Leaver, M. J., \& Tocher, D. R. 2009. Highly unsaturated fatty acid synthesis in Atlantic salmon: characterization of ELOVL5-and ELOVL2-like elongases. Mar Biotechnol, 11(5), 627-639. 
[23] Li, Y., Monroig, O., Zhang, L., Wang, S., Zheng, X., Dick, J. R. \& Tocher, D. R. 2010. Vertebrate fatty acyl desaturase with $\Delta 4$ activity. PNAS USA, 107(39), 16840-16845.

[24] Monroig, Ó., Wang, S., Zhang, L., You, C., Tocher, D. R., \& Li, Y. 2012. Elongation of long-chain fatty acids in rabbitfish Siganus canaliculatus: Cloning, functional characterisation and tissue distribution of Elovl5-and Elovl4-like elongases. Aquaculture, 350, 63-70.

[25] Soriguer, F., Serna, S., Valverde, E., Hernando, J., Martín-Reyes, A., Soriguer, M., \& Esteva, I. 1997. Lipid, protein, and calorie content of different Atlantic and Mediterranean fish, shellfish, and molluses commonly eaten in the south of Spain. Eur J Epidemiol, 13(4), 451-463.

[26] Baby, R. L., Hasan, I., Kabir, K. A., \& Naser, M. N. 2010. Nutrient analysis of some commercially important molluses of Bangladesh. J Sci Res, 2(2), 390-396.

[27] Liu, H., Zhang, H., Zheng, H., Wang, S., Guo, Z., \& Zhang, G. 2014. PUFA biosynthesis pathway in marine scallop Chlamys nobilis Reeve. J Agr Food Chem, 62(51), 12384-12391.

[28] Agaba, M. K., Tocher, D. R., Zheng, X., Dickson, C. A., Dick, J. R., \& Teale, A. J. 2005. Cloning and functional characterisation of polyunsaturated fatty acid elongases of marine and freshwater teleost fish. Comp Biochem Physiol. 142B (3), 342-352.

[29] Waldock, M. J., \& Holland, D. L. 1984. Fatty acid metabolism in young oysters, Crassostrea gigas: polyunsaturated fatty acids. Lipids, 19(5), 332-336.
[30] Viso, A.C., Marty, J.C., 1993. Fatty acids from 28 marine microalgae. Phytochemistry 34 (6), 1521-1533.

[31] Zhukova, N.V., Imbs, A.B., Yi, L.F., 1998. Diet-induced changes in lipid and fatty acid composition of Artemia salina. Comp Biochem. Physiol. 120B (3), 499-506.

[32] Zheng, X., Seiliez, I., Hastings, N., Tocher, D. R., Panserat, S., Dickson, C. A., \& Teale, A. J. 2004. Characterization and comparison of fatty acyl $\Delta 6$ desaturase cDNAs from freshwater and marine teleost fish species. Comp Bioch Physiol. Part B, 139(2), 269-279.

[33] Hastings, N.; Agaba, M. K.; Tocher, D. R.; Zheng, X.; Dickson, C. A.; Dick, J. R.; Teale, A. J. 2004. Molecular cloning and functional characterization of fatty acyl desaturase and elongase cDNAs involved in the production of eicosapentaenoic and docosahexaenoic acids from $\alpha$-linolenic acid in Atlantic salmon (Salmo salar). Mar Biotechnol, 6, 463-474.

[34] Tocher, D. R.; Zheng, X.; Schlechtriem, C.; Hastings, N.; Dick, J. R.; Teale, A. J. 2006. Highly unsaturated fatty acid synthesis in marine fish: Cloning, functional characterization, and nutritional regulation of fatty acyl $\Delta 6$ desaturase of Atlantic cod (Gadus morhua L.). Lipids 41, 1003-1016.

[35] Carmona-Antoñanzas, G.; Monroig, Ó.; Dick, J. R.; Davie, A.; Tocher, D. R. 2011. Biosynthesis of very long-chain fatty acids (C> 24) in Atlantic salmon: Cloning, functional characterisation, and tissue distribution of an Elovl4 elongase. Comp Biochem Physiol. 159B, 122-129. 